\title{
CORONAL MAGNETIC FIELDS ABOVE ACTIVE REGIONS
}

\author{
DAVID M. RUST
}

Sacramento Peak Observatory, Air Force Cambridge Research Laboratories, Sunspot, New Mex., U.S.A.

and

J.-RENÉ ROY

Dept. of Astronomy, University of Western Ontario, London, Ontario, Canada

\begin{abstract}
We have made detailed comparisons of coronal structure, as photographed at $\lambda 5303 \AA$, with magnetic lines of force, as computed from measurements of the longitudinal component of the underlying photospheric magnetic fields. Coronal fields were computed under the assumption that the space above the active regions is current-free. Out of 36 regions for which there exist both magnetic and coronal data at the Sacramento Peak Observatory, we found only four suitable for analysis. Using a light-pen attachment to a digital computer, we were able to choose lines of force whose footpoints in the photosphere best matched those of the lines of force which were suggested by local intensity variations in the corona. The lines of force thus computed give excellent agreement with the apparent height and shape of coronal fieldlines. In particular the magnetic field in the loop structures of 2 November 1969 and 18-19 November 1968 falls from several hundred gauss at 10000-20000 km above the photosphere to 2-20 G at heights of $100000-150000 \mathrm{~km}$. These computed values are in general agreement with other measurements. Spectra of the loops reveal that the direction of the lineof-sight component of the motion agrees with that anticipated from a comparison of the computed fieldline orientation and the observed motion in the plane of observation. We conclude that coronal magnetic fields above slowly changing active regions are nearly the same as the vacuum potential fields derived from underlying photospheric field sources (principally the larger sunspots in a region) and that calculations of this type may be relied upon to give the magnetic fields for studies of coronal dynamics.
\end{abstract}

Above active centers on the Sun, the solar corona is a luminous forest of loops, arcs and rays. Because of the strong magnetic fields known to exist in the underlying photosphere and because of the suggestive loops and arches, we expect that there are intense, complex magnetic fields in the corona over active centers. The only magnetograph measurements of these magnetic fields have been made by Harvey (1969), but because of the very low signal-to-noise ratio he encountered, he had to integrate the field measurements over areas much larger than that of typical coronal fine structure. Harvey's measurements indicated average coronal fields of a few gauss. Hyder (1964) inferred field intensities of 60 to $80 \mathrm{G}$ from his measurements of linear polarization in $\mathrm{H} \alpha$ loops at heights of between 5000 and $45000 \mathrm{~km}$ above the solar limb. Measurements in quiescent prominences, which, in contrast to loop prominences, are invisible in photographs taken with the coronal emission lines, may not be reliable indicators of the surrounding coronal fields. Correll et al. (1956), Correll and Roberts (1958), Warwick (1957), Bumba and Kleczek (1961) and Hyder (1966) have inferred either coronal field configurations or limits on coronal field intensities from the motions and shapes of solar prominences and flare sprays, but none of these indirect approaches allows one to specify the fields completely. Newkirk et al. (1968) and Altschuler and Newkirk (1969) used fictitious multipoles at the Sun's center to compute the complete magnetic field of the solar corona. They assumed that the corona is free of large scale 
currents and that the coronal field depends only upon the photospheric fields from which their multipoles are derived. They compared their computed field configuration with eclipse photographs of the white light corona and found good agreement between fieldlines and large scale structures such as streamers, domes over quiescent prominences, and arches linking active centers.

In 1963 Schmidt (1964) suggested that one may be able to neglect currents in the lower corona, i.e., between $10000 \mathrm{~km}$ and $150000 \mathrm{~km}$ above the limb, and compute the field directly from a potential derived from magnetograph measurements of the radial component of photospheric magnetic fields. Schmidt identified the measurements of the normal component of the field at disk center with fictitious magnetic monopoles. He then computed the vacuum field above the plane of observation, which he assumed to be flat. Harvey (1969) and Rust $(1966,1970)$ used the computer program devised by Schmidt to compute fields for comparison with coronal structure and with observed fields in prominences. In the work we are describing here, we extended Rust's and Harvey's studies to loop prominences and to some less distinct coronal structures above active regions. We used magnetograms from the Sacramento Peak non-sa-

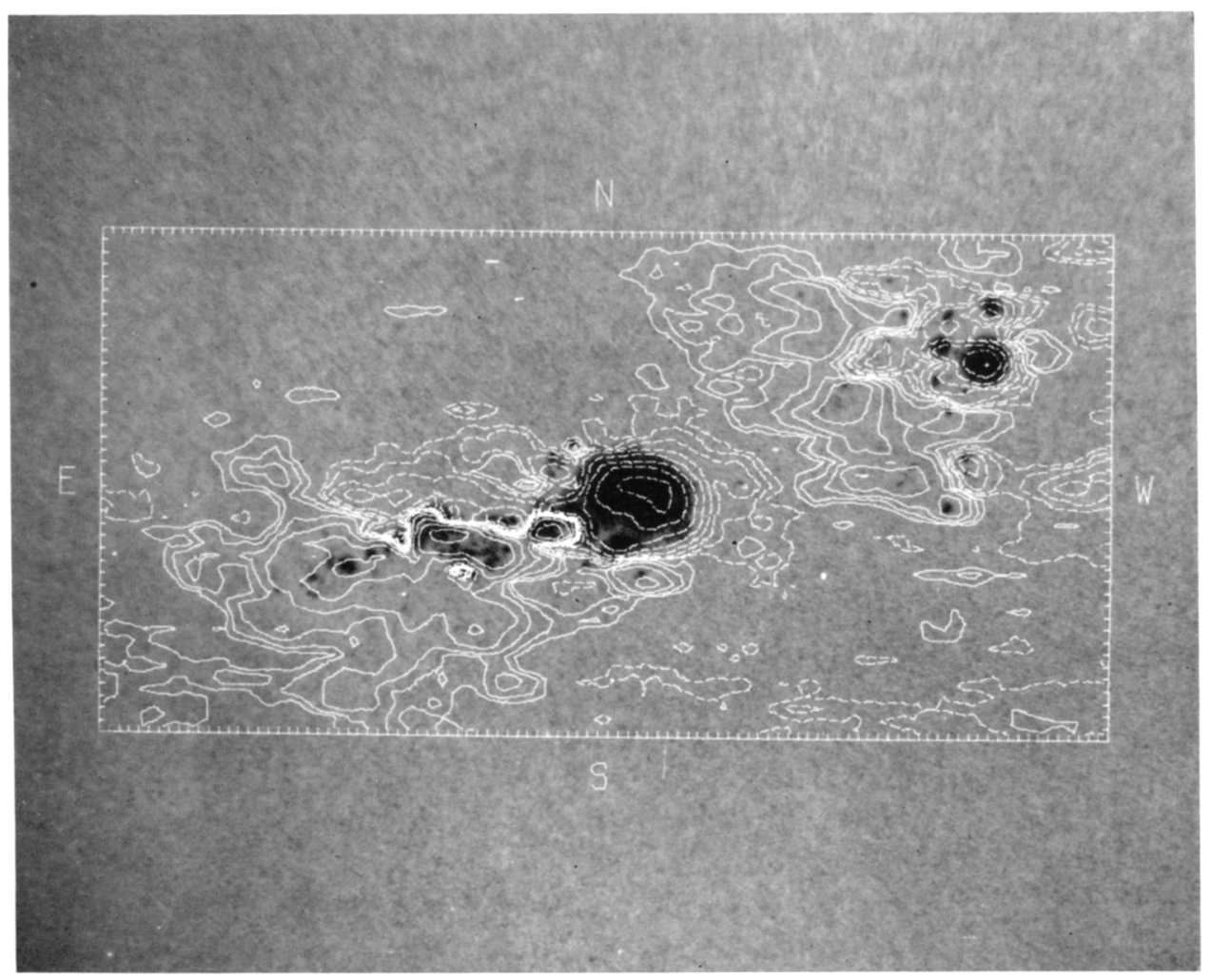

Fig. 1. Contours of longitudinal magnetic field intensity and the sunspots of Mt. Wilson spot regions 17533, 17544, and 17535. Time: 1500 UT, 24 October 1969. Dashed contours indicate negative fields, solid contours indicate positive fields. Contour levels are $\pm 10, \pm 20, \pm 40$, etc. 
turating Doppler-Zeeman Analyzer. The instrument was described by Dunn in an earlier session of this symposium. Monochromatic pictures of the corona at $\lambda 5303 \AA$ and spectra and slit-jaw photographs in $\mathrm{H} \alpha$ obtained at the Observatory during the years 1968 and 1969 were available for this study. Dr. Robert Howard provided us with copies of the visual sunspot field measurements from the Mount Wilson Observatory for comparison with the measurements from the Doppler-Zeeman Analyzer.

From the rich store of data available to us, we found 36 active centers for which there exist disk-center magnetic maps and green-line photographs of the corona as

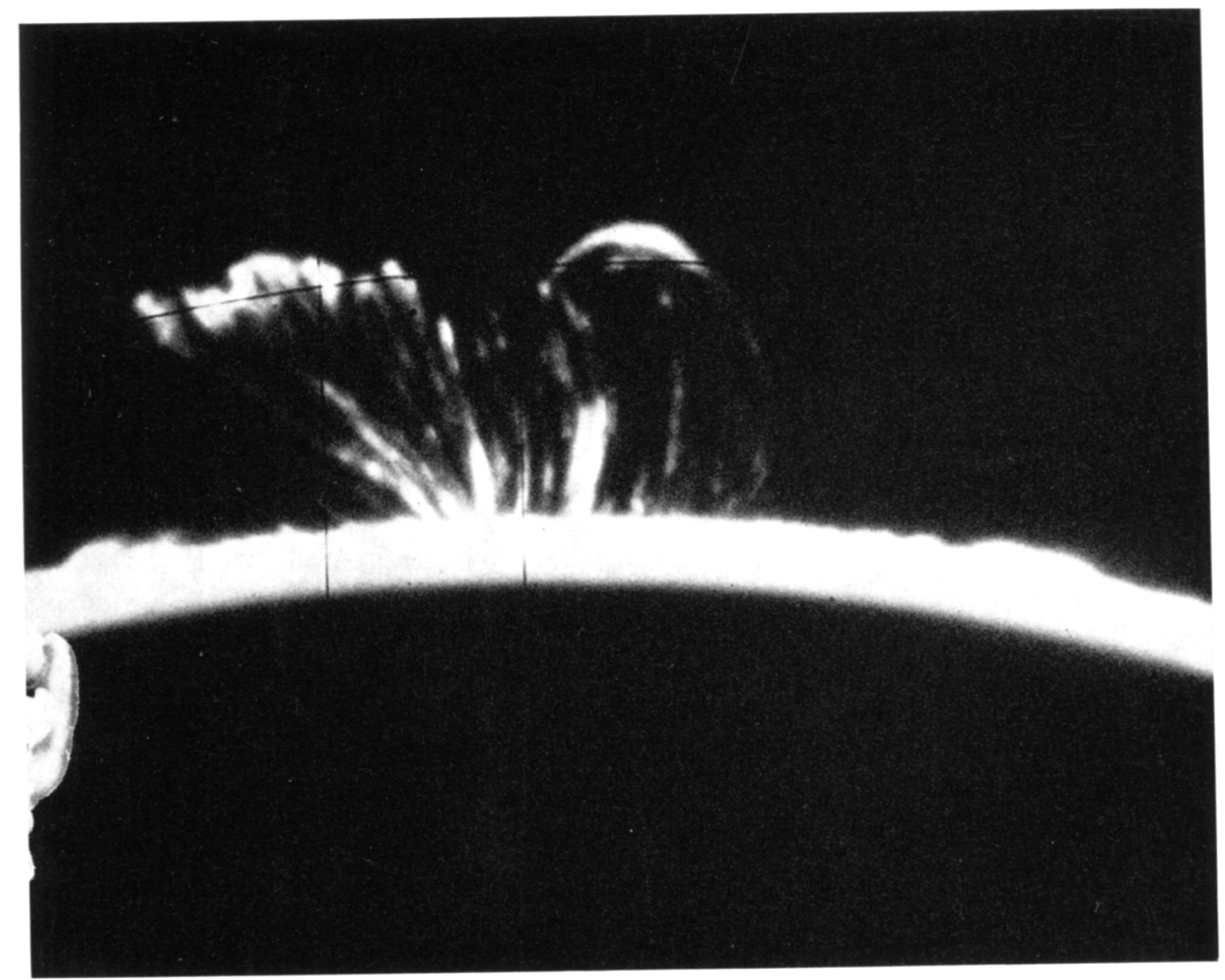

Fig. 2. $\mathrm{H} \alpha$ loops seen above the region of Figure 1 , when it was $12^{\circ}$ beyond the west limb on 2 November 1969. Feet of the loops are hidden behind the chromosphere.

the center passed over the limb. The first step in our analysis was to eliminate from further consideration all centers that underwent substantial evolution from disk center to limb passage. Furthermore, the quality of some magnetograms was too poor for quantitative work, and other magnetograms were rejected because they did not include important regions of magnetic flux near the active center under study. We were left with only four relatively stable active centers for analysis. We have found that, in each of these cases, the potential fields derived from photospheric observations 


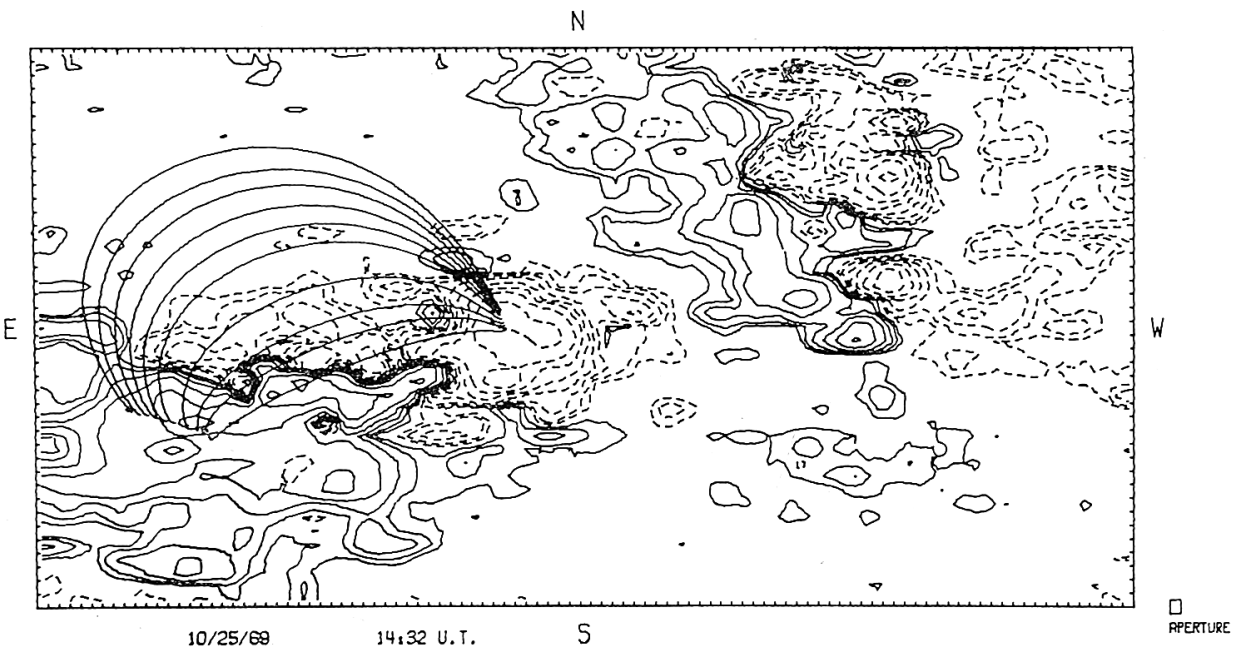

Fig. 3. Contours of longitudinal magnetic field one day later than Figure 1. The smooth, thin lines are the fieldlines which give the best fit to the loops seen in the preceding figure. Notice how the leftmost footpoints of the fieldlines form a ribbon parallel to the dividing line between regions of opposite polarity. The other footpoints concentrate in a small area of the strong negative fields in the big sunspot (see Figure 1).

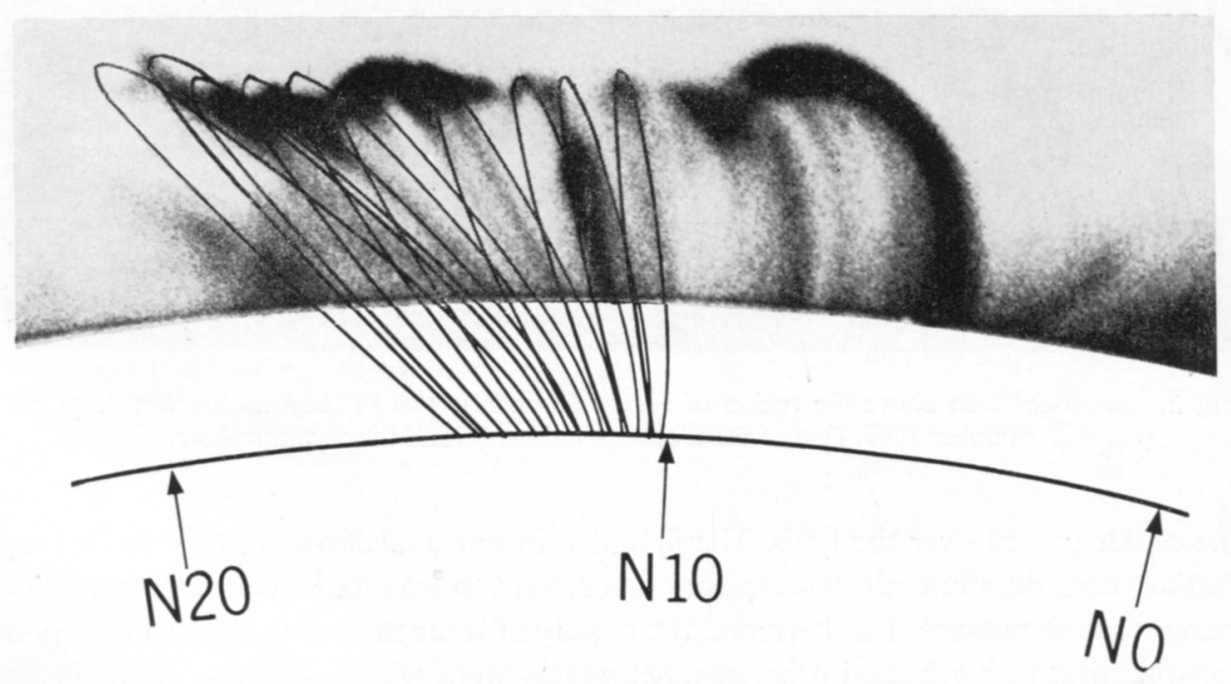

Fig. 4. Computed fieldlines for the loops of 2 November 1969. The loops are seen here on an overocculted negative photograph of the corona at $\lambda 5303 \AA$. The lower arc indicates the level of the chromosphere. These coronal loops are nearly identical to the $\mathrm{H} \alpha$ loops of Figure 2, which were photographed at about the same time. 
match the coronal structure very closely. No data which met the selection criteria were eliminated from study once we started to compute the magnetic fields.

Figure 1 shows the magnetic-field contours in Mount Wilson spot regions Numbers 17533,17544 and 17535 . The lowest contour level is $10 \mathrm{G}$ and the highest field measured was in the large spot at the center and was equal to $1800 \mathrm{G}$. Visual measurements of line splitting, made at Mount Wilson, indicated a maximum field of $2100 \mathrm{G}$. After several comparisons of this sort we concluded that, although our magnetic observations were made in the temperature-sensitive line of iron at $5250 \AA$, the magnetic fields recorded by the Doppler-Zeeman Analyzer are not less than about $70 \%$ of the true fields. When the photograph of Figure 1 was taken the largest region in the picture was $20^{\circ}$ east of the central meridian. On the following day, 25 October 1969, we obtained another magnetogram. The indicated fields were very similar to those seen in Figure 1. When the easternmost of the regions shown was $12^{\circ}$ over the west limb, there occurred in the region a very large flare, producing the largest X-ray burst of the year and a spectacular series of loops, as shown in the hydrogen-alpha photograph (Figure 2). This region has a fairly featureless and apparently unchanging corona on the 17 th and 18 th of October, when it was on the east limb, and on the 30th of October and the 1st of November, as it passed over the west limb. The loops photographed in Figure 2 provide us with a distinct structure to compare with computed fieldlines. Figure 3 shows the fieldlines that fit the loops. We would like to remark here that, from the work of Bruzek (1964), we don't think that the appearance of loops where there was previously an amorphous structure indicates a change in the coronal fields. Loops only show where material ejected during the flare is condensing in the corona. Furthermore, it is well known that there are no changes in the major sunspots or magnetic fields at the time of a flare. Whatever changes occur in association with flares take the form of minor adjustments in the field intensity or in the position of small magnetic features, such as may be seen next to the large spot in Figure 1. The shapes of the fieldlines shown here are little affected by any but the largest magnetic poles in the region. Figure 4 shows the loops as they appeared at $\lambda 5303 \AA$. The computed fieldlines shown are the same as those in the previous figure, but they are seen at the position they took when the region was $12^{\circ}$ over the west limb at about $1500 \mathrm{UT}$ on 2 November 1969. Both sets of loops are $108000 \mathrm{~km}$ above the underlying photospheric surface. The field intensity at the loop tops varies from $6 \mathrm{G}$ to $16 \mathrm{G}$ and increases to hundreds of gauss at about $25000 \mathrm{~km}$ above the large spot. The flare associated with these loops was a major producer of protons, and we note that the footpoints of the loops penetrate the umbra of the major spot (Figure 3). Bruzek has shown that the footpoints of loop systems fall along the two ribbons of large $\mathrm{H} \alpha$ flares. Since proton flares are known for the fact that the $\mathrm{H} \alpha$ emission of one of the ribbons penetrates the umbra of major spots, the appearance of the computed footpoints in the umbra further confirms the correctness of the computed fields.

Examination of the spectra of the loops shows that the legs of the loops which are more inclined to the solar surface are blue-shifted along the upper half of the loops. Since we are looking at the loops nearly edge-on, the more inclined, blue-shifted loops 
must be the closest to us. The computed fieldlines also show the same mixture of inclinations and the more inclined loops are also closer to the observer. A side view of the computed loops suggests that if the material in the loops is moving along the fieldlines everywhere with the same velocity, one should see red shifts in the rearward legs of the loops which are greater than the blue shifts coming from the forward legs. An examination of the spectra confirmed that this is the case.

In two details the computed loops do not fit the observed loops. First, the computed

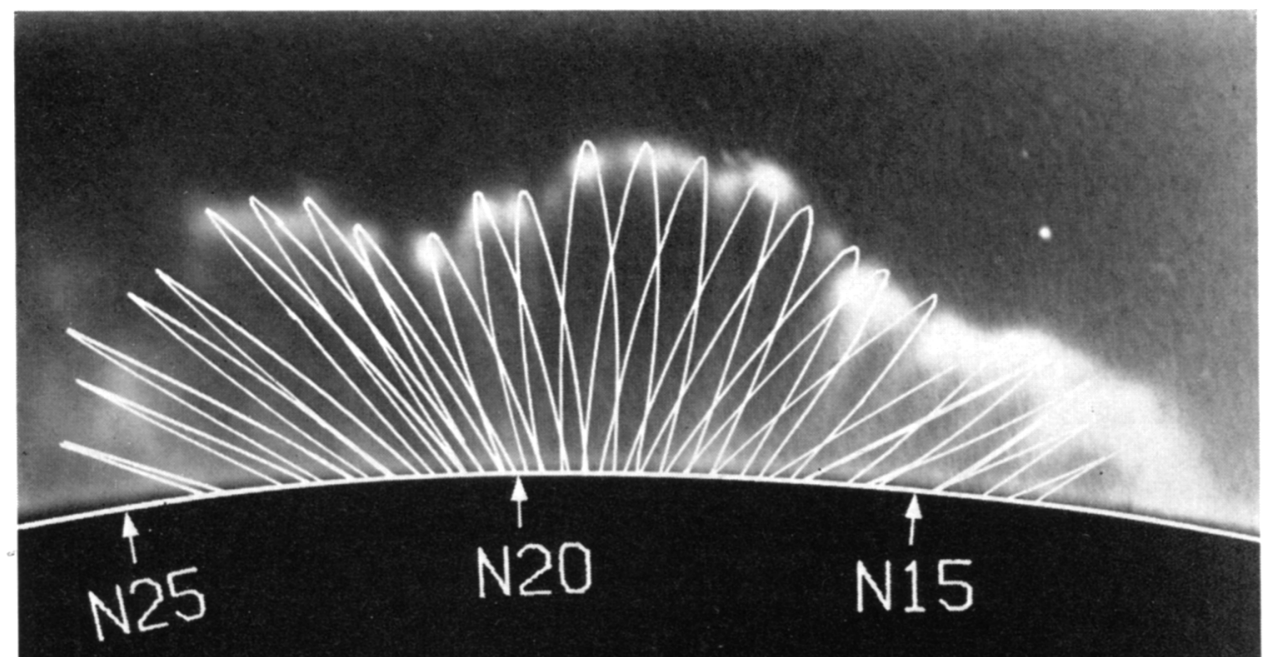

COMPUTED FIELDLINES

\section{NOVEMBER 1968}

\section{WEST LIMB}

Fig. 5. Loops seen in the coronal $\lambda 5303 \AA$ emission with computed fieldlines superposed upon them. Numbers indicate heliographic coordinates.

fieldlines lean slightly more to the side. This may probably be explained as an edge effect, arising from the fact that outside the plane of observation, we have assumed that there is no field in the photosphere. The exact configuration of leaning loops is sensitive to small magnetic features near the edges of the active center. The second detail in which our computed loops do not fit the observations concerns our inability to fit the large loop on the right. We think there may have been some rotation of the following spots about the leader - this is often observed. A change of this sort between 
the disk observation of 25 October and the coronal observation of 2 November could produce the needed field configuration.

Figure 5 shows the computed fieldlines and observed loops for 18 November 1968. The fit is excellent everywhere, except for a small difference in the inclination of the loops, as observed and as calculated, on the edge of the frame. The loops are about $90000 \mathrm{~km}$ high at the center. The field there equals $11 \mathrm{G}$, while the field intensity at the tops of the side loops is $4 \mathrm{G}$. The loops observed on this date as well as the loops shown in the earlier figures are typical in height and configuration. They are two-tothree times the height of the loops measured by Hyder. When lower, but similar, loops

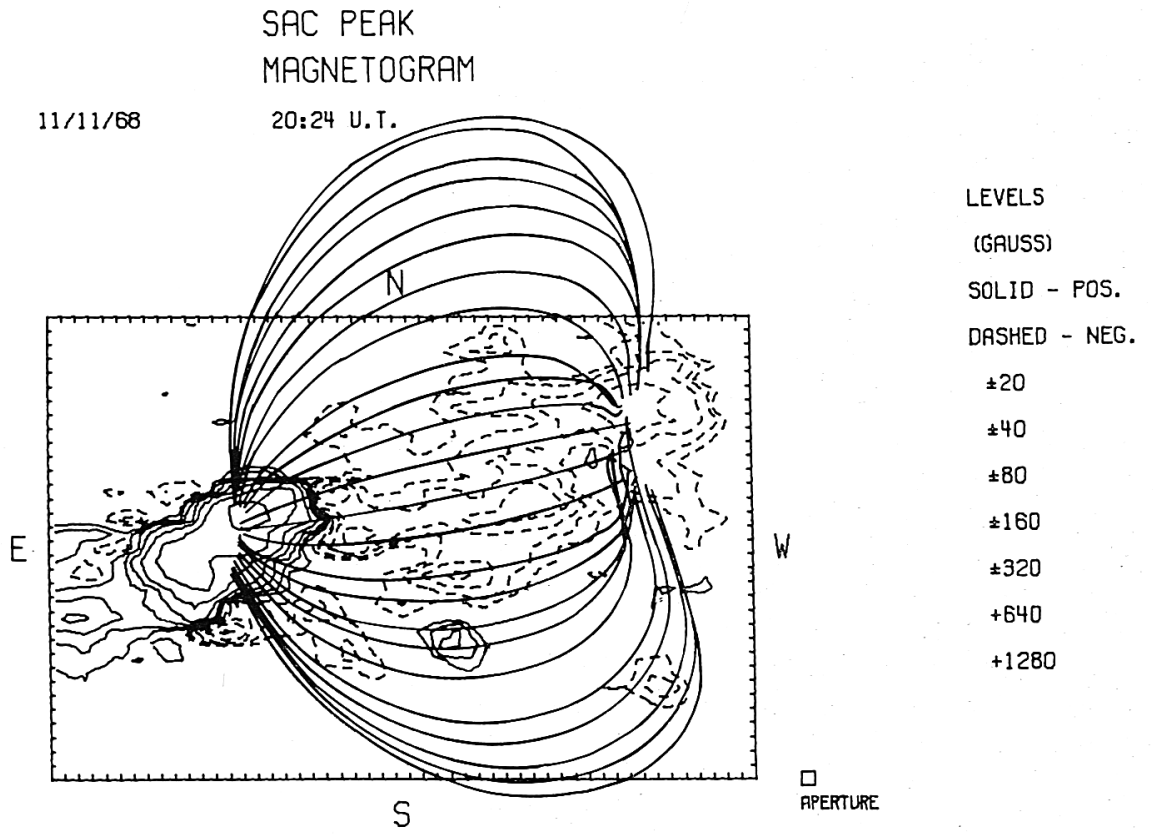

Fig. 6. Fieldlines of Figure 5 as seen from directly above the active center.

are computed for the regions studied here, the resultant field intensities at the loop tops agree well with those measured by Hyder.

Figure 6 shows the loops as seen from directly above the active center. At the top of the picture, the loops swing out above the region included in the magnetogram. If there had been some positive field there, instead of the zero field we assumed, those lines of force wouldn't have been so inclined toward the surface, and they would have fit the observations better. We feel that this failure to fit the observed structures exactly derives from this limitation in the measurements, and it is not due to currents in the corona. Notice that the lines of force fall in two ribbons which flank the line dividing magnetic polarities. We expect that these ribbons reveal the location of the $\mathrm{H} \alpha$ flare associated with the loops. 
At the lower half of Figure 6, the fieldlines pass over two small concentrations of field. The effect of these fields is to add several inflection points along the edge loops. A large-scale motion picture of the region taken in the $D_{3}$ emission of helium shows that falling material apparently follows fieldlines with inflection points similar to those we found in our calculations. Inflection points of this sort may imitate twisted fieldlines and suggest the presence of currents. We feel that current-free fields are adequate to explain the apparent twisting of the fields observed here.

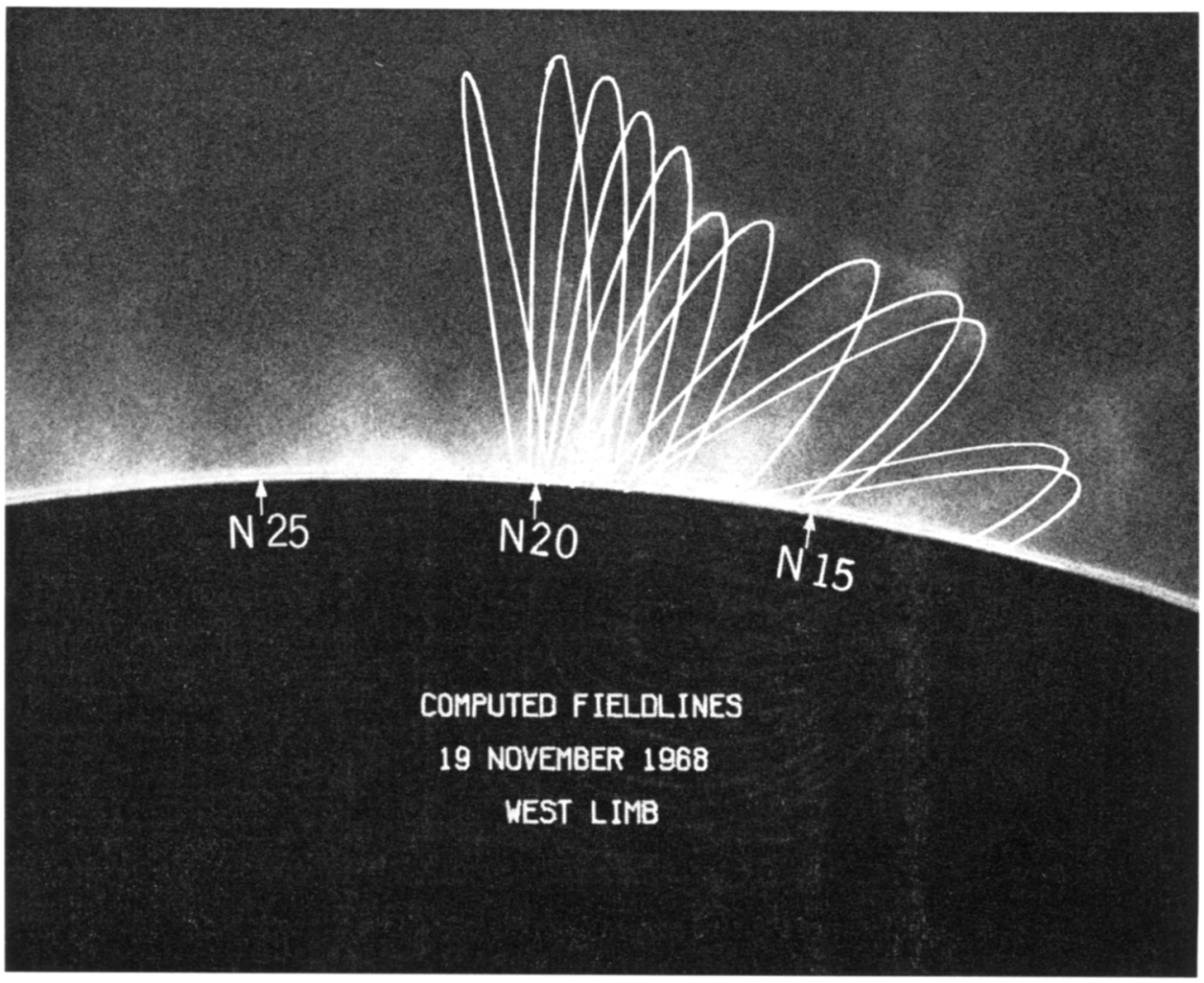

Fig. 7. Computed fieldlines and observed coronal structure for 19 November 1968. The brilliant loops of the previous day (Figure 5) have faded to a higher and barely detectable veil.

Figure 7 shows how the loops appeared on the next day. They were higher and much fainter. This time they were visible only in the corona. Except for some edge effects, the computed loops fit the observations very well. At the loop tops, from $80000 \mathrm{~km}$ to $145000 \mathrm{~km}$ high, the field intensity is 2 to $4 \mathrm{G}$. The footpoints form two ribbons in the photosphere somewhat farther apart than those for the brighter, lower loops of the previous day.

Spectra of the loops show more blue-shifted material than red-shifted material. A view of the loops face-on readily explains this result since both legs of the loops are 
inclined toward the observer. Only along a small segment of the visible loops does downfalling matter have a component of motion away from the observer.

So far, we have discussed only loop-prominence observations. Figure 8 illustrates the success enjoyed in fitting vacuum fields to the coronal condensation of 27 March 1969. Since it is impossible to tell from the coronal pictures whether the fieldlines are closed or open at the top, we calculated both kinds of lines. The difference in open and closed lines is only a few seconds of arc in the position of the footpoints away from the center-line of the underlying fields. The fields at 70000 to $100000 \mathrm{~km}$ are 7 to $4 \mathrm{G}$ respectively. Another case of fitting to a coronal condensation is illustrated in Figure 9. As in the previous case, the indistinctness of the corona limits the impressiveness of the correlation.

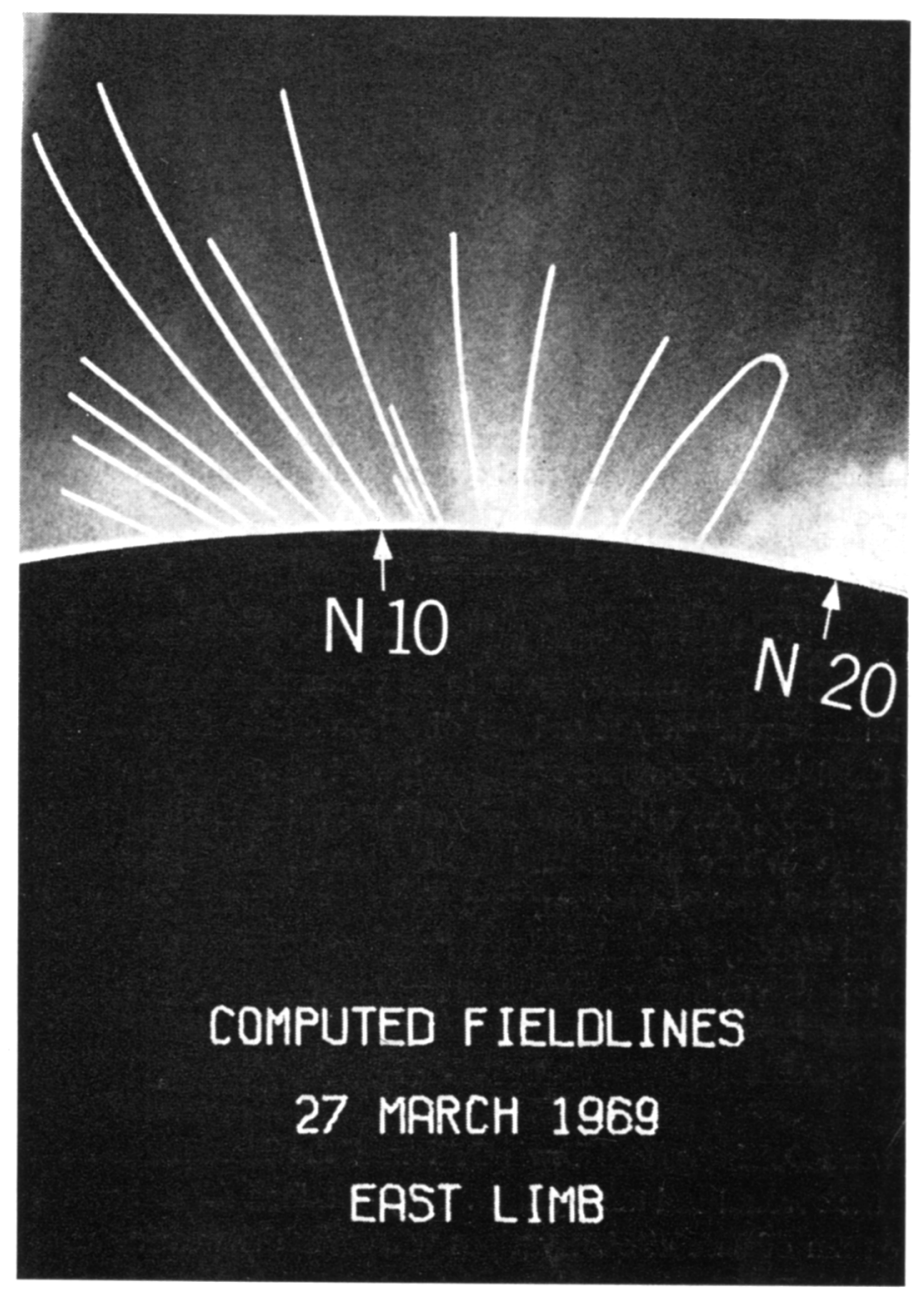

Fig. 8. Computed fieldlines and coronal rays above an east limb active center. 


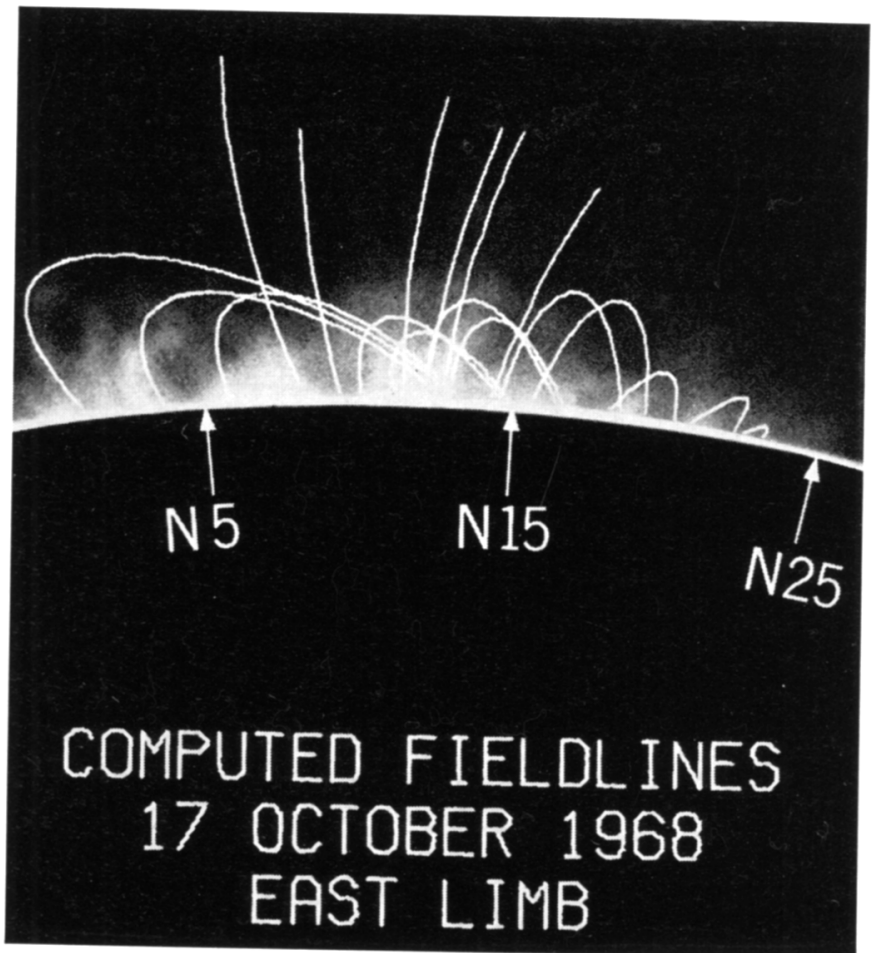

Fig. 9. Less distinct coronal structure and computed fieldlines for an east limb condensation. The original negatives reveal a background of rays paralleling the computed fieldlines.

\section{Summary}

We have found that vacuum potential fields, computed from disk-center observations of slowly changing active centers, seem to fit coronal structures very closely. The computed fieldlines apparently reveal the direction of the fields in the corona. The magnitude of the fields we compute agrees well with observations and probably is too low by only about $30 \%$ due to the difficulty of measuring the true photospheric fields with the 5250 line. Where movies and spectra are available, it should be possible, with the aid of the Schmidt program, to derive complete three-dimensional maps of the magnetic and velocity fields in the corona above active centers. We plan to extend our study into this area to better understand the dynamics of the corona.

\section{Acknowledgements}

We thank Howard DeMastus and Lewis Gilliam of the Sacramento Peak Observatory for lending us the coronal and the $D_{3}$ films: They obtained these excellent photographs through a program of careful observations whenever the weather would allow. We are also grateful to Dr Charles Hyder and to Dr William Wagner for the use of the $\mathrm{H} \alpha$ pictures and for the spectra of the loops. 


\section{References}

Altschuler, M. D. and Newkirk, G. A.: 1969, Solar Phys. 9, 131.

Bruzek, A.: 1964, Astrophys. J. 140, 746.

Bumba, V. and Kleczek, J.: 1961, Observatory 81, 141.

Correll, M., Hazen, M. and Bahng, J.: 1956, Astrophys. J. 124, 597.

Correll, M. and Roberts, W. O.: 1958, Astrophys. J. 127, 726.

Harvey, J. W.: 1969, Thesis, University of Colorado.

Hyder, C. L.: 1964, Astrophys. J. 140, 817.

Hyder, C. L.: 1966, Z. Astrophys. 63, 78.

Newkirk, G. A., Altschuler, M. D., and Harvey, J. W.: 1968, in K. O. Kiepenheuer (ed.), 'Structure and Development of Solar Active Regions', IAU Symp. 35, 379.

Rust, D. M.: 1966, Thesis, University of Colorado.

Rust, D. M.: 1970, Astrophys. J. 160, 315

Schmidt, H. U.: 1964, in AAS-NASA Symp. Phys. Solar Flares, (ed. by W. N. Ness), NASA SP-50, p.107.

Warwick, J. W.: 1957, Astrophys. J. 125, 811.

\section{Discussion}

Leighton: How does the Sun decide which lines of force to condense matter upon, and how do you choose which lines to compute?

Rust: I don't know the answer to the first question. As for the way I choose the lines to draw, I take my cue from the Sun. There is an infinite number of possible lines to draw, but all but a few of them are irrelevant to the comparisons shown.

Severny: My question has much in common with that of Dr. Leighton. So I will phrase the question by asking how many 'sources' or equivalent dipoles do you include in your calculations of lines of force? I found that your calculated field has only two equivalent \pm and - 'poles' or places of divergence and convergence of lines of force. Meanwhile on the maps you showed there are many magnetic poles of equal strength in between these particular places. Generally the problem of calculation of lines of force in such circumstances is equivalent to $n$-body problem with dipole-type interaction. In the Crimea we usually include in the computer program not less than four or five equivalent dipoles to find the actual behavior of lines of force and this behavior appears to be very peculiar sometimes, more complex than you showed.

Rust: At each point along the field lines, we take into account the influence of all 5000 or so points of the magnetogram. If we drew the lines of force which lie close to the photosphere, we would get more complex shapes, but the loops are simple structures because they are so high.

Schatten: Rather than being critical of Dr. Rust's choice of the footpoints of the field lines, I am impressed that there are some field lines which agree as well as they do! Furthermore, in some of my field calculations I observe the highest closed arches above active regions to be about $1 / 10-1 / 5$ of a solar radius. This occurs often when an active region is surrounded by a strong background field of one polarity. It is possible that these field lines that you have chosen are the highest closed lines and that coronal material is 'raining' down on these lines. Were these the biggest closed field lines in your calculation?

Rust: No. There were other, higher lines that closed.

Jordan: You say that your calculated loop structure agrees with that observed in the active regions. However, you have compared only with the Fexiv line. The results from the 1970 eclipse (Speer $e t$ al., 1970, Nature) show that the loop structure is quite different in lines formed in different temperature regions.

Rust: The green-line observations are the only ones available to us, at least on a regular basis. It would be interesting, but also difficult, to gather the data for the more complete comparison you mentioned. 